\title{
Ictiofauna acompanhante na pesca artesanal do camarão sete-barbas (Xiphopenaeus kroyeri) no litoral sul do Brasil
}

\author{
Mário Cesar Sedrez, Joaquim Olinto Branco', Felipe Freitas Júnior', \\ Herbert Silva Monteiro ${ }^{1}$ \& Edison Barbieri ${ }^{2,3}$ \\ ${ }^{1}$ Centro de Ciências Tecnológicas da Terra e do Mar, Universidade do Vale do Itajaí - UNIVALI, \\ Rua Uruguai, 458, CP 360, CEP 88302-202, Itajaí, SC, Brasil \\ ${ }^{2}$ Instituto de Pesca, Agência Paulista de Tecnologia dos Agronegócios - APTA, \\ Secretaria de Agricultura e Abastecimento do Estado de São Paulo - SAA/SP, Av. Prof. Besnard, s/n, \\ CEP 11990-000, Cananéia, SP, Brasil \\ ${ }^{3}$ Autor para correspondência: Edison Barbieri, e-mail: edisonbarbieri@yahoo.com.br
}

SEDREZ, M.C., BRANCO, J.O., FREITAS JUNIOR, F.; MONTEIRO, H.S. \& BARBIERI, E. Ichthyofauna bycatch of sea-bob shrimp (Xiphopenaeus kroyeri) fishing in the town of Porto Belo, SC, Brazil. Biota Neotrop. 13(1): http://www.biotaneotropica.org.br/v13n1/en/abstract?inventory+bn00313012013

\begin{abstract}
The activity of artisanal fishing for the sea-bob shrimp, using the method of motorized dragnet with rings, has been practised on the Santa Catarina coast, where it generates jobs and income, and helps keep the traditional Azorean cultural tradition alive, since 1960. With the aim of analyzing the quality and quantity of the ichthyofauna bycatch, two trawls were carried out per isobath (10-20-30 m), seasonally, between November 2009 and August 2010 (i.e. summer, spring and winter), for 20 minutes each, at an average speed of two knots, and the bottom water temperatures and salinities recorded. The captures totalled 10868 fish $(208.34 \mathrm{~kg})$, distributed among 31 families and 62 species, with a fish/shrimp ratio of 5.19/1 kg. The most abundant family was Sciaenidae $(86.13 \%)$, followed by Batrachoididae $(2.70 \%)$ and Trichiuridae $(2.44 \%)$. The diversity and evenness indices presented similar patterns of variation, the highest values occurring in spring at $30 \mathrm{~m}$ and the lowest in winter at $20 \mathrm{~m}$. Cluster analysis led to the creation of four groups; one of them small and dominated by the Sciaenidae Stellifer brasiliensis, S. rastrifer, Paralonchurus brasiliensis, Isopisthus parvipinnis and Larimus breviceps. The young of this and other species, which are of little or no commercial value, along with tiny shrimps and other macroinvertebrates that enter the dragnets, are thrown back into the sea, usually dead. Besides the negative impact on the food chain, this continued practice could exacerbate the situation of artisanal fishermen even further, with the gradual, future decline of these resources.
\end{abstract}

Keywords: Xiphopenaeus kroyeri, demersal fish, impacts of fishing, trawling, Porto Belo.

SEDREZ, M.C., BRANCO, J.O., FREITAS JUNIOR, F., MONTEIRO, H.S. \& BARBIERI, E. Ictiofauna acompanhante na pesca artesanal do camarão sete barbas (Xiphopenaeus kroyeri) no litoral sul do Brasil. Biota Neotrop. 13(1): http://www.biotaneotropica.org.br/v13n1/pt/abstract?inventory+bn00313012013

Resumo: A pesca artesanal dirigida ao camarão sete-barbas (Xiphopenaeus kroyeri), usando o método de arrasto motorizado com portas, tem sido praticada na costa de Santa Catarina, onde ele gera emprego e renda, e ajuda a manter a tradição cultural tradicional açoriana viva, desde 1960. Com o objectivo de analisar a qualidade e a quantidade das capturas acidentais da ictiofauna, efetuou-se sazonalmente, dois arrastos por isóbata (10-20-30 m) entre novembro/2009 e agosto/2010, duração de $20 \mathrm{~min} /$ cada, velocidade média dois nós e, registrados temperaturas e salinidades da água de fundo. As capturas totalizaram 10868 peixe $(208.34 \mathrm{~kg})$, distribuídos em 31 famílias e 62 espécies, com uma proporção de peixe/camarão de 5,19/1 kg. A família mais abundante foi Sciaenidae (86.13\%), seguido por Batrachoididae (2.70\%) e Trichiuridae (2,44\%). Os índices de diversidade e equabilidade apresentaram padrões similares de variação, os maiores valores ocorreram na Primavera na profundidade de $30 \mathrm{~m}$ e a mais baixa no inverno, a $20 \mathrm{~m}$. A análise de Cluster formou quatro agrupamentos, sendo um deles, pequeno e dominante de Sciaenidae Stellifer brasiliensis, S. rastrifer, Paralonchurus brasiliensis, Isopisthus parvipinnis e Larimus breviceps. Os exemplares jovens dessas espécies e de outras, com pouco ou sem valor comercial, camarões miúdos e demais macro-invertebrados acompanhantes capturados são descartados ao mar, geralmente mortos. Além do impacto nas cadeias tróficas, essa prática contínua, pode agravar ainda mais a situação do pescador artesanal com o declínio desses recursos nas capturas futuras

Palavras-chave: Xiphopenaeus kroyeri, peixes demersais, impactos da pesca, pesca de arrasto, Porto Belo. 


\section{Introdução}

O Estado de Santa Catarina é o principal produtor de pescado no Brasil (Brasil 2011), legado da cultura açoriana, que contribuiu expressivamente para o desenvolvimento da pesca artesanal do camarão sete-barbas (Xiphopenaeus kroyeri), além do próprio desenvolvimento da pesca industrial nas comunidades litorâneas catarinenses (Branco et al. 2006).

Devido à baixa seletividade da pesca de arrasto, há captura de grande contingente da fauna bentônica e demersal, agrupados como fauna acompanhante ou "by-catch" (Alverson et al. 1994). Os peixes demersais ou ictiofauna constituem o grupo predominante e de maior interesse da fauna acompanhante (Branco \& Verani 2006b). A parte desembarcada é caracterizada por indivíduos com valor comercial e a rejeitada, por indivíduos sem valor econômico ou por exemplares pequenos de espécies valiosas (Graça-Lopes et al. 2002).

Os produtos pesqueiros apresentam grande relevância na alimentação humana, já que contribuem com $16,0 \%$ da proteína animal consumida no mundo (Rocha \& Rocha 2010). Logo, a pesca artesanal está entre as principais fontes geradoras dessa proteína, visto que só em Santa Catarina, existem aproximadamente 25 mil pescadores artesanais (Empresa... 2010). Por outro lado, em virtude da baixa seletividade da pesca de arrasto, esta pode ser considerada um dos maiores problemas atuais na utilização dos recursos marinhos, pois promove grande impacto ambiental e desperdício de alimento (Graça-Lopes et al. 2002).

Nesse contexto, o presente estudo tem como objetivo analisar quali e quantitativamente a ictiofauna acompanhante da pesca de arrasto artesanal do camarão sete-barbas em Porto Belo/SC, o impacto da pesca de arrasto tanto espacial como temporal, sendo que a partir dos dados sobre o impacto pode-se verificar a necessidade de manejo e quais medidas devem ser implementadas.

\section{Material e Métodos}

As coletas foram realizadas em regime sazonal, na área de atuação da frota artesanal do camarão sete-barbas de Porto Belo, SC (2709' 12" S e 48 33' 43” W) (Instituto... 2011), entre os anos de 2009 a 2010, nas isóbatas de 10, 20 e $30 \mathrm{~m}$ (Figura 1), durante o dia, nas seguintes estações do ano: primavera (outubro/2009), verão (janeiro/2010), outono (abril/2010) e inverno (julho/2010).

Foram realizados dois arrastos de $20 \mathrm{~min} /$ isóbata, utilizando-se uma embarcação da frota local, denominada "baleeira", equipada com duas redes de arrasto com portas, malha de $3,0 \mathrm{~cm}$ na manga e corpo e, 2,0 cm no ensacador, com velocidade aproximada de dois

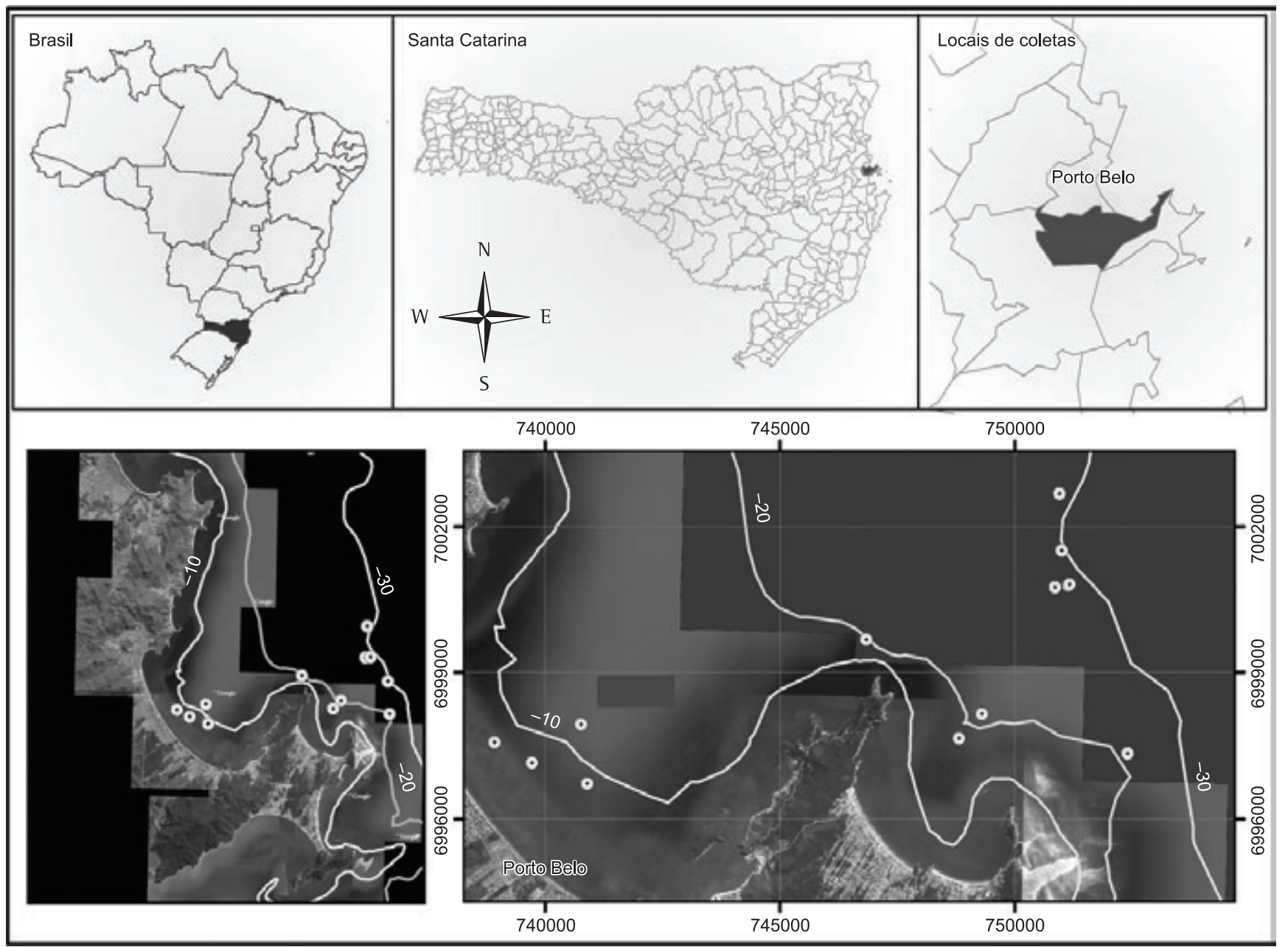

Figura 1. Localização da área de estudo da pesca artesanal do camarão sete-barbas (Xiphopenaeus kroyeri), quando utilizando os pesqueiros de Porto Belo, SC. As linhas indicam as isóbatas de 10,20 e $30 \mathrm{~m}$ e os pontos os locais mais utilizados pela pesca.

Figure 1. Location of the study area of artisanal fishing of sea-bob shrimp (Xiphopenaeus kroyeri), using the fishing grounds of Porto Belo, SC. The lines indicate the isobaths of 10,20 and 30 meters and the most used for fishing. 
nós (Branco 2005). Durante as coletas, foram registradas no início de cada arrasto, as temperaturas e as salinidades (fundo e superfície).

O produto de cada arrasto foi separado a bordo, etiquetado, conservado em caixas de isopor com gelo e transportado ao laboratório. A ictiofauna acompanhante foi identificada de acordo com as chaves taxonômicas de Topp \& Hoff (1972), Figueiredo (1977, 1980), Soares (1978), Figueiredo \& Menezes (1978, 2000) e Menezes \& Figueiredo $(1980,1985)$. A nomenclatura dos táxons seguiu o padrão internacional, utilizando-se a base de dados online da FishBase (2011).

Conforme a ocorrência das espécies nas coletas, foram classificadas em muito frequentes (MF) apareceram em $70 \% \mathrm{ou}$ mais dos arrastos, as frequentes (F) entre $40 \%$ e $69,99 \%$, as pouco frequentes (PF) entre $20 \%$ e $39,99 \%$ e as ocasionais (OC) em 19,99\% ou menos (Graça-Lopes et al. 1993).

A captura por unidade de esforço (CPUE) em número de exemplares $(\mathrm{N} / \mathrm{h})$ e biomassa $(\mathrm{kg} / \mathrm{h})$ da ictiofauna acompanhante, foi calculada pela média dos arrastos na isóbata por estação do ano, multiplicados por três, equivalendo à uma hora de pesca. Também foi utilizado o somatório das CPUE do camarão sete-barbas, fauna acompanhante e lixo (material inorgânico) nas quatro estações do ano, para determinada a participação relativa da ictiofauna em relação aos demais grupos integrantes dessa modalidade de pesca.

A análise de variância paramétrica One-Way ANOVA (Sokal \& Rohlf 1995), através do software Instat, foi utilizada para se verificar a existência de diferenças significativas entre as CPUE da ictiofauna acompanhante por isóbata e estações do ano. Foram testados quanto à homogeneidade da variância (teste de Bartlett) e a normalidade da distribuição (prova de Kolmorov-Smirnov). O contraste das médias, através do teste Tuckey-Kramer, foi aplicado na ocorrência de diferenças significativas para indicar a fonte causadora das variações (Branco \& Verani 2006a)

A estrutura das populações foi avaliada através dos índices de diversidade de Shannon (H') e equitabilidade de Pielou (J') (Ludwig \& Reynolds 1988).

Pela análise de agrupamento estabeleceram-se as medidas de semelhanças entre os pares de espécies, pelo coeficiente de distância Euclidiana e os agrupamentos, pelo método de Ward, implementados no software Statistica 6 (Branco \& Verani 2006a). Em função da distribuição contagiosa típica de peixes, houve a padronização dos dados através da transformação logarítmica $\log (\mathrm{x}+1)$ (Colvocoresses \& Musick 1984). As espécies de ocorrência ocasional foram eliminadas das análises de agrupamento (Branco \& Verani 2006b).

\section{Resultados}

\section{Variáveis ambientais}

A temperatura da água de fundo apresentou valores mais elevados na primavera nas diferentes isóbatas e menores no inverno nos $30 \mathrm{~m}$ (Tabela 1). A média anual variou entre $20,94{ }^{\circ} \mathrm{C} \pm 2,83,20,69 \pm 1,91$ e 19,61 $\pm 1,27$, respectivamente nas isóbatas de 10,20 e $30 \mathrm{~m}$, sem diferenças significativas entre essas $(F=0,9918 ; p>0,05)$, entretanto foram diferentes sazonalmente $(\mathrm{F}=23,7430 ; \mathrm{p}<0,05)$.

Para a salinidade da água de fundo (Tabela 1), os maiores valores ocorreram nos $30 \mathrm{~m}$, durante a primavera e os menores em $10 \mathrm{~m}$, no inverno, sendo a média anual entre 32,26 $\pm 0,97(10 \mathrm{~m})$, $33,24, \pm 1,23(20 \mathrm{~m})$ e $33,10 \pm 1,08(30 \mathrm{~m})$, sem diferenças entre as isóbatas $(F=1,6820 ; p>0,05)$, porém, diferiram significativamente entre as estações $(\mathrm{F}=9,0020 ; \mathrm{p}<0,05)$.

\section{Composição das capturas}

A Ictiofauna acompanhante contribuiu com $68,49 \%$ da biomassa capturada $(208,34 \mathrm{~kg})$, seguida da espécie-alvo camarão sete-barbas $(13,20 \%)$, Echinodermata (2,64\%), Crustacea (9,20\%), Mollusca $(0,27 \%)$, Cnidaria $(3,51 \%)$ e lixo inorgânico (2,69\%) (Figura 1a).

Para cada quilograma de camarão sete-barbas, em média foram capturados 5,19 de ictiofauna. (Figura 2a).

\section{Ictiofauna}

Durante o período amostral foram capturados 10868 exemplares, agrupados em 31 famílias, 51 gêneros e 62 espécies, sendo 6,45\% de Condrichthyes e 93,55\% de Actinopterygii (Tabela 2). Das espécies, 17 foram comuns as três isóbatas, 3,23\% consideradas muito frequentes, $16,13 \%$ frequentes, $27,42 \%$ pouco frequentes e $53,23 \%$ ocasionais (Tabela 2 ).

\section{Contribuições das famílias e espécies}

Os Sciaenidae contribuíram com $86,13 \%$ dos peixes amostrados, seguido dos Batrachoididae (2,70\%), Trichiuridae (2,44\%), Pristigasteridae (1,91\%), Cynoglossidae (1,09\%), Carangidae $(0,98 \%)$, sendo que as outras 22 famílias em conjunto contribuíram $\operatorname{com}(4,44 \%)$ da abundância capturada (Figura 2b).

A espécie Stellifer brasiliensis reuniu $27,77 \%$ e $19,96 \%$ respectivamente, dos exemplares e biomassa dos peixes capturados, seguida por S. rastrifer, com $16,58 \%$ e $15,06 \%$ e Paralonchurus brasiliensis, com $14,69 \%$ e $19,72 \%$ (Tabela 2 ).

Tabela 1. Temperatura $\left({ }^{\circ} \mathrm{C}\right)$ e salinidade médias da água de fundo por isóbata e estação do ano, na pesca artesanal do camarão sete-barbas em Porto Belo, SC. Table 1. Temperature $\left({ }^{\circ} \mathrm{C}\right)$ and salinity average of the deep water in relation the isobath and station of the year, in artisanal fishing for the sea-bob shrimp in Porto Belo, SC, Brazil.

\begin{tabular}{|c|c|c|c|c|c|}
\hline \multicolumn{6}{|c|}{$10 \mathrm{~m}$} \\
\hline Variáveis & Primavera & Verão & Outono & Inverno & Média anual \\
\hline Temperatura & 25,00 & 20,75 & 19,00 & 19,00 & $20,94 \pm 2,83$ \\
\hline Salinidade & 33,05 & 33,15 & 31,50 & 31,35 & $32,26 \pm 0,97$ \\
\hline \multicolumn{6}{|c|}{$20 \mathrm{~m}$} \\
\hline Temperatura & 23,00 & 21,50 & 19,00 & 19,25 & $20,69 \pm 1,91$ \\
\hline Salinidade & 32,95 & 35,05 & 32,45 & 32,50 & $33,24 \pm 123$ \\
\hline \multicolumn{6}{|c|}{$30 \mathrm{~m}$} \\
\hline Temperatura & 21,50 & 19,20 & 19,00 & 18,75 & $19,61 \pm 1,27$ \\
\hline Salinidade & 34,05 & 34,00 & 32,00 & 32,35 & $33,10 \pm 1,08$ \\
\hline
\end{tabular}




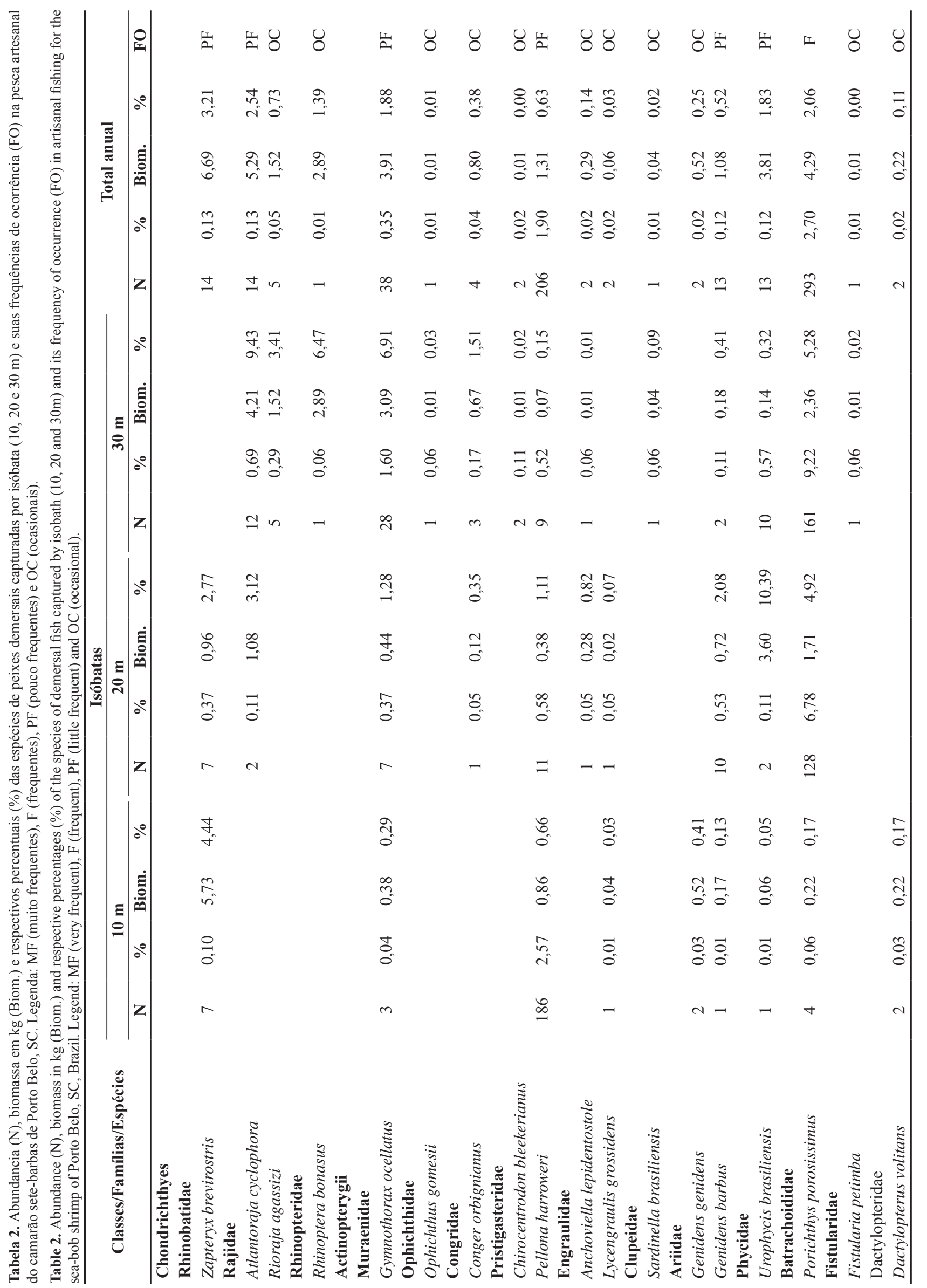




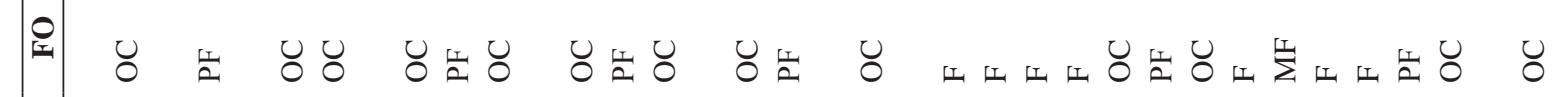

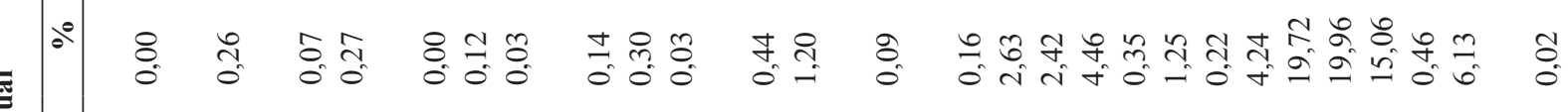

至

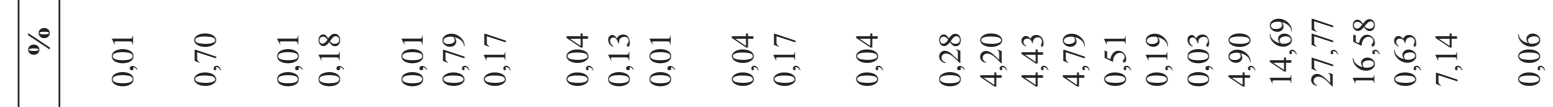

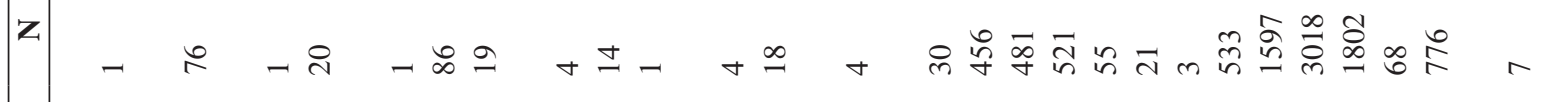

$\therefore$ :

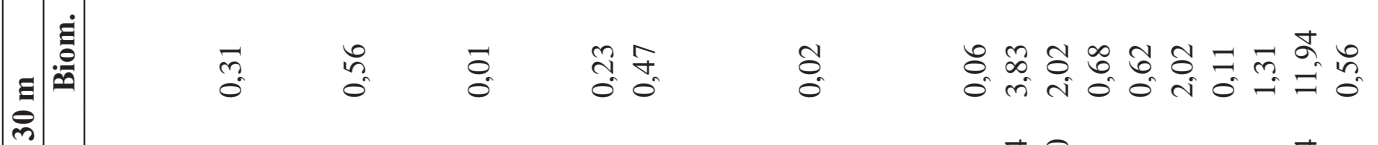

$\begin{array}{llllll}0 & 0 & 0 & 0 & 0 & 0\end{array}$

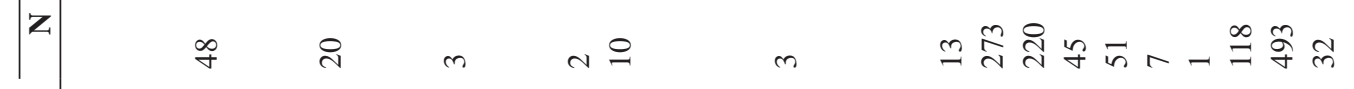

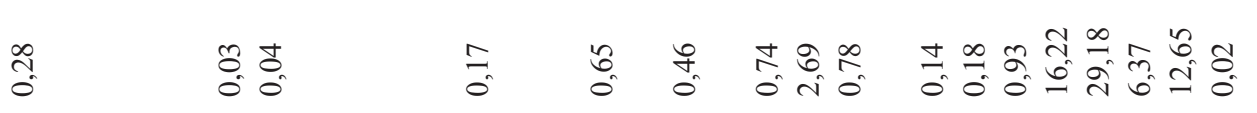

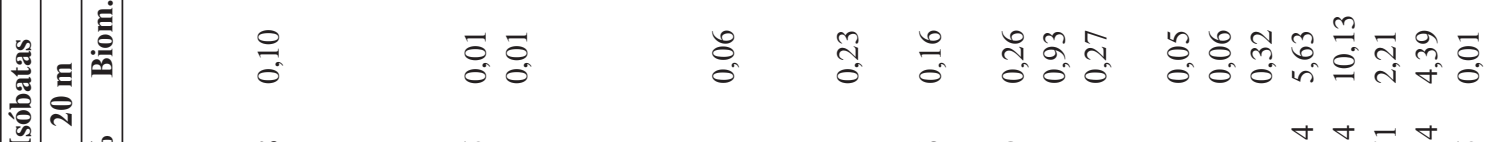

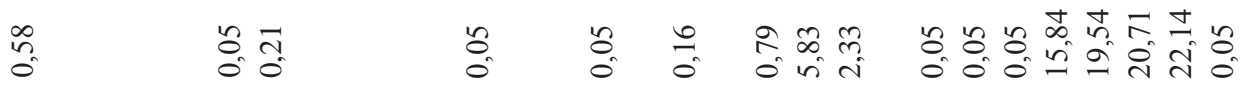

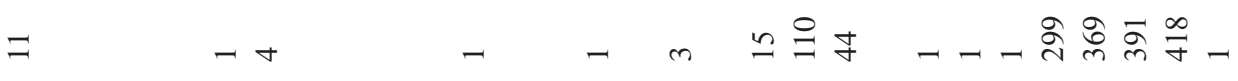

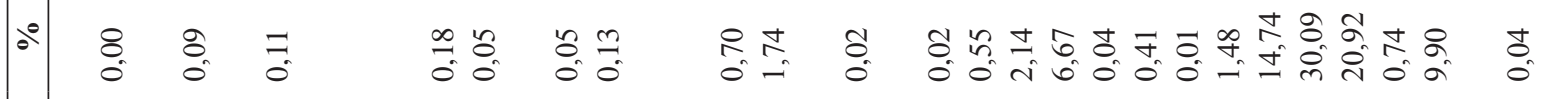

I

$\therefore$

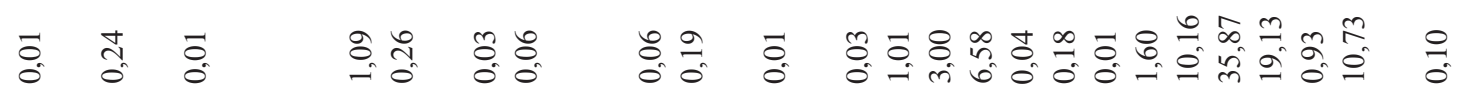

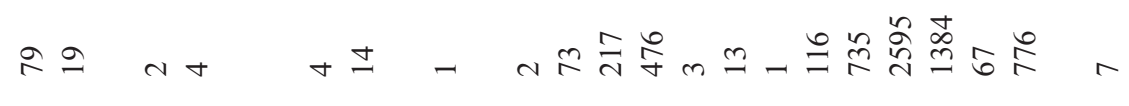


Sedrez, M.C. et al.

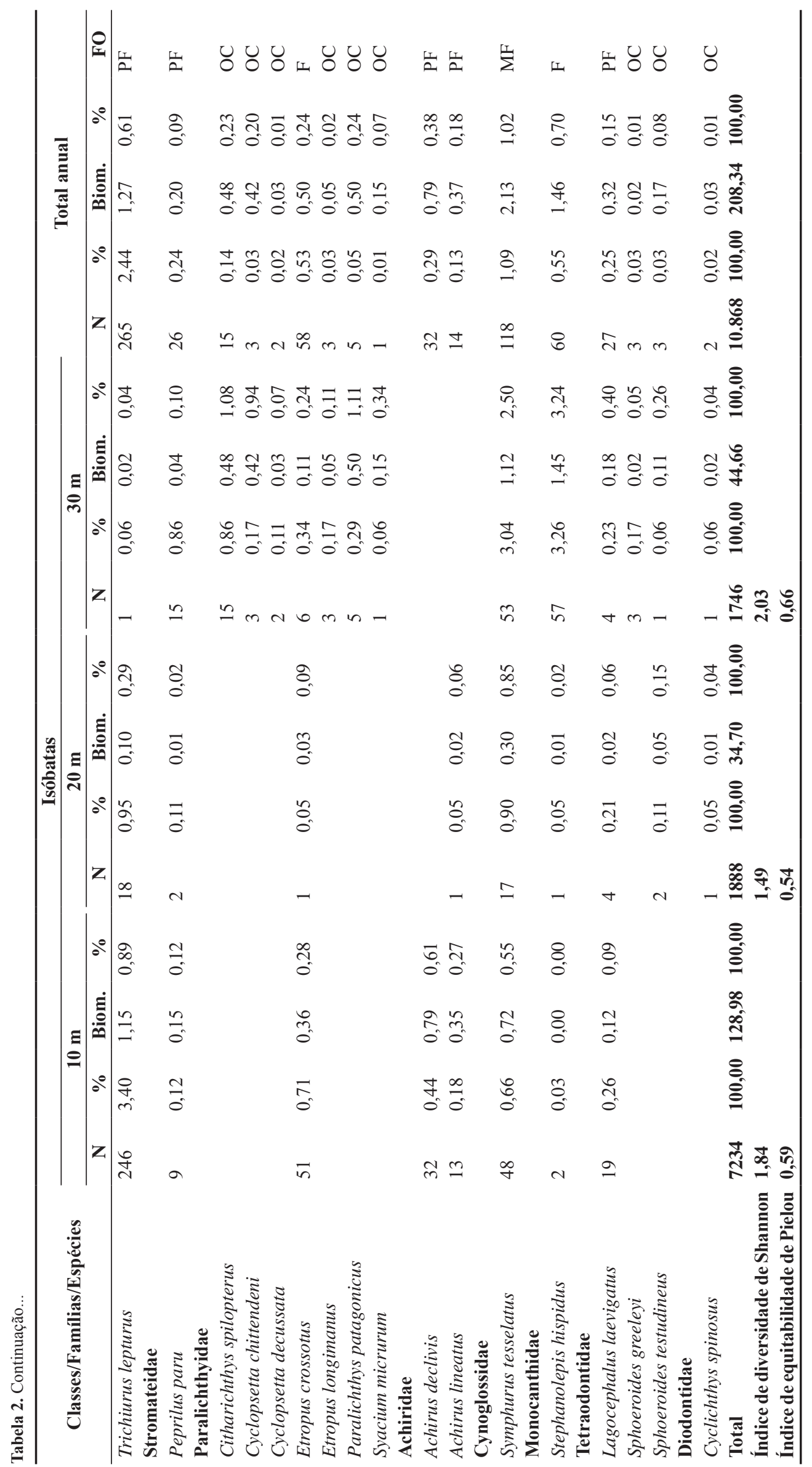




\section{Captura por unidade de esforço}

Foram observadas oscilações sazonais nas CPUE médias em abundância e biomassa dos exemplares (Figura 2), sendo que no verão ocorreram as maiores abundâncias e no inverno as menores nos $20 \mathrm{~m}$, seguidos da primavera e outono (30 m) (Figura 3a); enquanto que as maiores capturas em biomassa foram registradas durante as estações de inverno, verão, primavera e outono nos $10 \mathrm{~m}$ e as menores no outono (30 m), primavera e inverno (20 m) (Figura 3b).

Em geral, a abundância de exemplares reduziu com o aumento da profundidade, não apresentando diferenças significativas entre as estações do ano $(\mathrm{F}=1,0980 ; \mathrm{p}>0,05)$, porém, mostraram-se significativamente diferentes entre as isóbatas $(F=8,9940 ; p<0,05)$. Já a biomassa alternou-se entre os 10,30 e $20 \mathrm{~m}$, sem diferenças significativas sazonais $(\mathrm{F}=0,4633 ; \mathrm{p}>0,05)$, sendo significantes nas isóbatas $(\mathrm{F}=6,4200 ; \mathrm{p}<0,05)$ (Tabela 2).
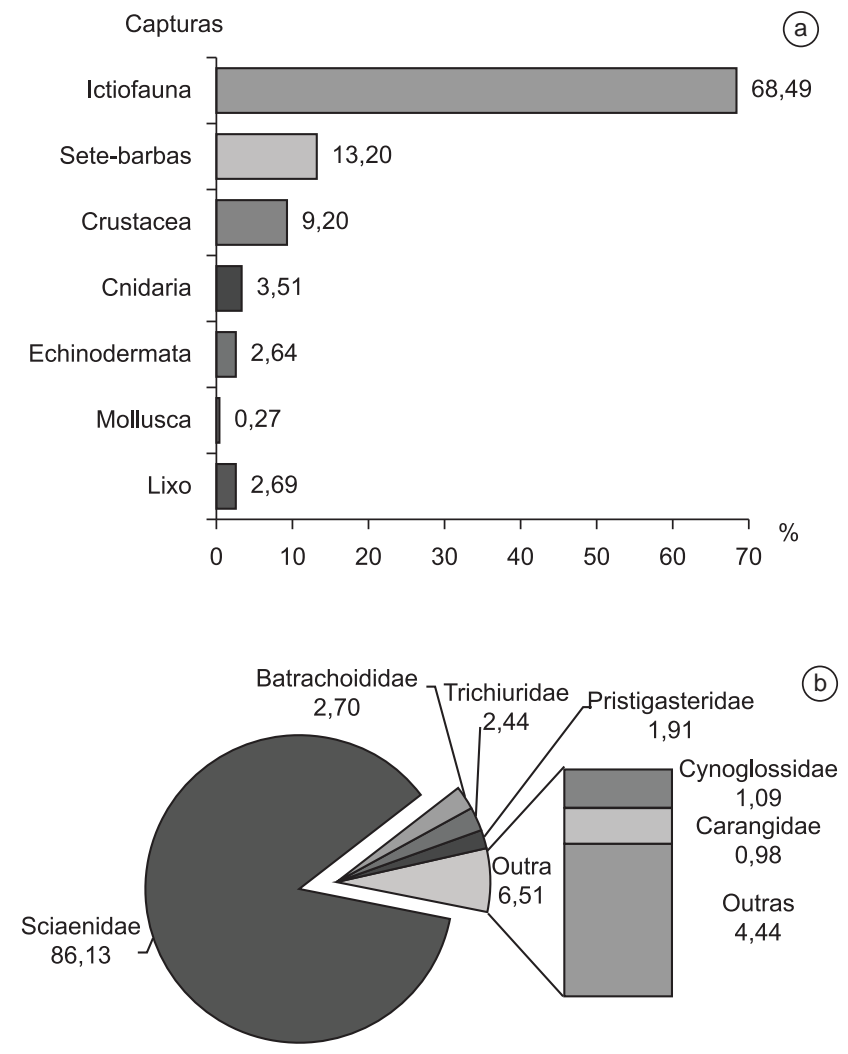

Figura 2. Composição relativa em biomassa (\%) nas capturas (a) e contribuição em número de exemplares (\%) das famílias da ictiofauna (b).

Figure 2. Biomass relative composition (\%) in the catch (a) and in number of copies contribution (\%) of the families of the Ichthyofauna (b).

\section{Constância das espécies}

Nas isóbatas de 10, 20 e 30 m ocorreram respectivamente, 41, 36 e 26 espécies de peixes demersais, com pequenas oscilações sazonais, iniciando com 39 espécies na primavera, chegando ao máximo de 43 no verão e queda progressiva entre o outono (34) e inverno (32).

\section{Diversidade e equitabilidade}

O índice de diversidade de Shannon (H') apresentou os maiores valores na primavera $(2,43)$ nos $30 \mathrm{~m}$ e menores no inverno $(0,91)$ em $20 \mathrm{~m}$, com média anual de 1,84 $\pm 0,26(10 \mathrm{~m}), 1,49 \pm 0,43(20 \mathrm{~m})$ e 2,03 $\pm 0,30$ (30 m) (Tabela 3).

A equitabilidade seguiu a tendência de variação da diversidade entre as estações e isóbatas, oscilando entre 0,77 e 0,44, com médias anuais de $0,59 \pm 0,05 ; 0,54 \pm 0,08$ e $0,66 \pm 0,09$, respectivamente, nos 10,20 e $30 \mathrm{~m}$ (Tabela 3 ).

\section{Associação faunística}

A análise de Cluster aplicada as 29 espécies capturadas, com frequência de ocorrência em mais de quatro coletas anuais (Tabela 2), formou quatro agrupamentos com uma distância de junção em nível “10" (Figura 4), onde o grupo I reuniu o maior número de espécies, com oito pouco frequentes $(\mathrm{PF})$ e uma frequente $(\mathrm{F})$, porém, com baixa abundância; o II apresentou sete PF e uma F, ambas com pouca participação nas coletas; o III agrupou seis espécies, entre as 12 mais abundantes anualmente, sendo cinco F, duas PF e uma muito frequente (MF), porém, pouco abundante; e o IV reuniu as espécies mais abundantes, com quatro $\mathrm{F}$ e uma MF.

\section{Discussão}

As oscilações observadas na temperatura e salinidade da água de fundo nas coletas, podem ser atribuídas a pluviosidade, contribuições do rio Tijucas (Carvalho et al. 1998) e a presença sazonal da ACAS (Águas Centrais do Atlântico Sul) entre a primavera e verão, retraindo-se no inverno, deixando as temperaturas homogêneas e mais baixas na zona costeira (Matsuura 1986, Carvalho et al. 1998, Soares-Gomes \& Figueiredo 2002).

Carvalho et al. (1998) constataram um gradiente decrescente de temperatura partindo do Norte catarinense para o Sul, abaixo de $22^{\circ} \mathrm{C}$, próximo à Ilha do Arvoredo, na primavera/verão, indicando o afloramento da ressurgência costeira ao sul de Porto Belo, favorecida pelos ventos fortes do quadrante norte, porém, durante o outono/ inverno, a coluna d'água mantém-se homogênea em decorrência de subsidência, relacionada ao vento sul.

O bycatch elevado na pesca artesanal do camarão sete-barbas ficou dentro do esperado para a área de trabalho (Branco \& Verani 2006a), superando consideravelmente a biomassa de camarões capturados, em cerca de 5,2 vezes, com as maiores capturas incidindo sobre a ictiofauna e as menores nos Echinodermata e Mollusca. Essa

Tabela 3. Variação sazonal dos índices de Shannon (H') e Pielou (J'), nas isóbatas de 10, 20 e 30 m, na pesca artesanal do camarão sete-barbas de Porto Belo, SC. Table 3. Seasonal variation of the indices of Shannon (H') and Pielou (J'), in the isobath of 10, 20 and $30 \mathrm{~m}$, in artisanal fishing for the sea-bob shrimp of Porto Belo, SC, Brazil.

\begin{tabular}{|c|c|c|c|c|c|c|}
\hline \multirow{2}{*}{$\begin{array}{c}\text { Estações / } \\
\text { Isóbatas }\end{array}$} & \multicolumn{3}{|c|}{$\mathbf{H}^{\prime}$} & \multicolumn{3}{|c|}{ J' } \\
\hline & 10 & 20 & 30 & 10 & 20 & 30 \\
\hline Primavera & 1,80 & 1,44 & 2,43 & 0,56 & 0,49 & 0,77 \\
\hline Verão & 2,17 & 1,93 & 2,01 & 0,65 & 0,63 & 0,62 \\
\hline Outono & 1,84 & 1,66 & 1,99 & 0,60 & 0,60 & 0,70 \\
\hline Inverno & 1,54 & 0,91 & 1,69 & 0,53 & 0,44 & 0,57 \\
\hline Média anual & $1,84 \pm 0,26$ & $1,49 \pm 0,43$ & $2,03 \pm 0,30$ & $0,59 \pm 0,05$ & $0,54 \pm 0,09$ & $0,66 \pm 0,09$ \\
\hline
\end{tabular}


(a)
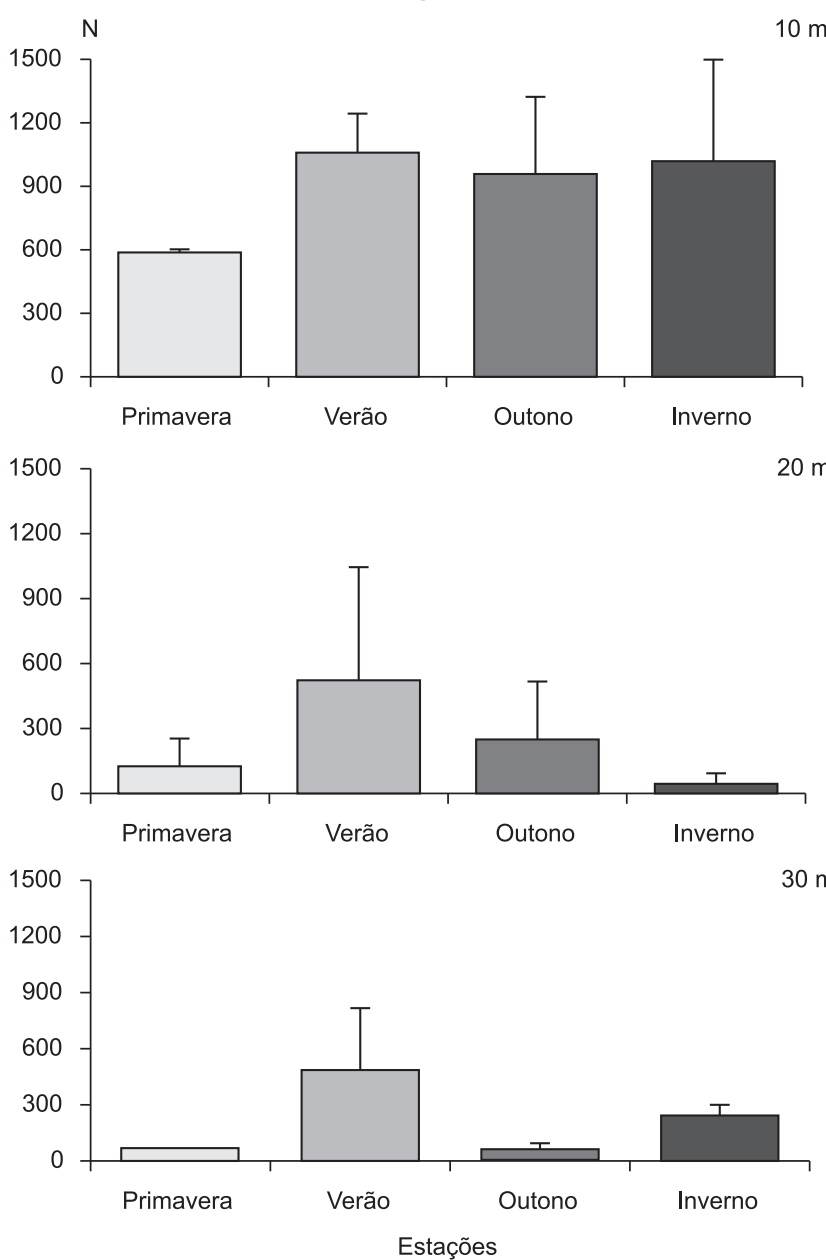

(b)
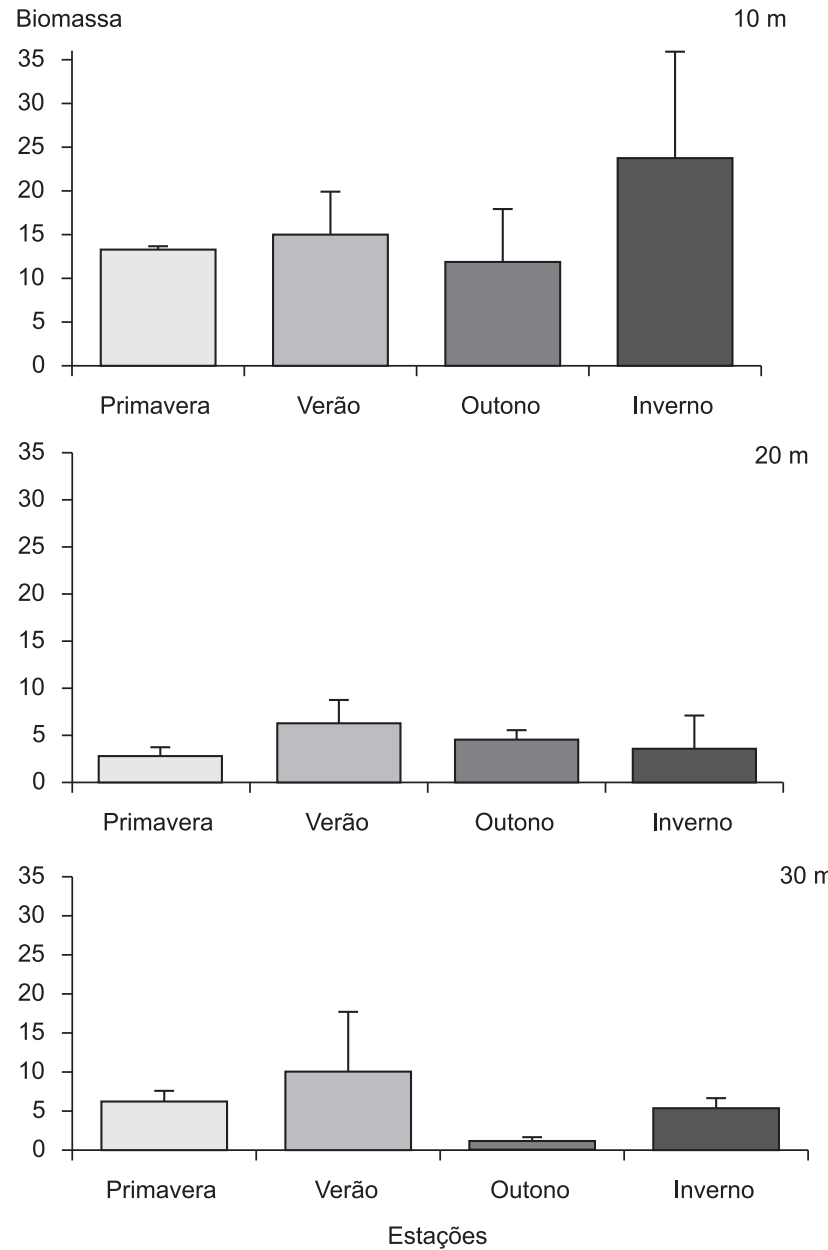

Figura 3. Variação sazonal da CPUE em abundância (a) e biomassa em kg (b) da ictiofauna acompanhante, nas Isóbatas de 10 m, 20 m e 30 m, na pesca artesanal do camarão sete-barbas (Xiphopenaeus kroyeri), durante 2009 e 2010 em Porto Belo, SC. As barras verticais representam o erro da média.

Figure 3. Seasonal variation of the CPUE in abundance (a) and biomass in $\mathrm{kg}$ (b) of ictiofauna accompanying, in the isobath of $10 \mathrm{~m}$, $20 \mathrm{~m}$ and $30 \mathrm{~m}$ in artisanal fishing for the sea-bob shrimp (Xiphopenaeus kroyeri), during 2009 and 2010 in Porto Belo, SC, Brazil. The vertical bars represent the error of the average.

tendência foi registrada em diversos estudos no litoral catarinense (Bail \& Branco 2003, Branco \& Verani 2006a, b, Bail et al. 2009, Bernardes Júnior et al. 2011) e norte de São Paulo (Graça-Lopes 2002a).

As proporções obtidas entre as biomassas de camarões setebarbas e ictiofauna acompanhante nos arrastos, tem seguem o padrão mundial variando de 5:1 em águas temperadas até 10:1 em tropicais (Slavin 1983); 9,3:1 no Brasil (Alverson et al. 1994); no litoral norte de São Paulo entre 1,26:1 (Graça-Lopes et al. 2002) e 3:1 a 9:1 (Souza et al. 2008), de 0,57:1 (Cattani et al. 2011) a 3:1 (Pinheiro \& Martins 2009) no Paraná. Em Santa Catarina, oscilam entre 1:1 a 8:1 na Penha (Branco \& Verani 2006a) a 3:1 na Praia de Gravatá/ Brava, Navegantes/Itajaí (Bail et al. 2009), provavelmente em função da maior abundância e incidência dos arrastos sobre os exemplares jovens da ictiofauna em algumas áreas de pesca.

A ictiofauna acompanhante vem sendo o componente mais capturado pela pesca artesanal de camarão, mas ainda é pouco aproveitada, apesar do interesse econômico em certas espécies (Diegues 1983, Branco 2005, Branco \& Verani 2006a). Essa situação demonstra a eficiência na captura da espécie-alvo e baixa seletiva, especialmente nos peixes demersais (Alverson et al. 1994, Rocha \&
Rossi-Wongtschowski 1998, Branco \& Verani 2006a, Souza et al. 2008, Bail et al. 2009, Bernardes Júnior et al. 2011).

Há uma grande variação entre o número de famílias e espécies na ictiofauna capturada ao longo do litoral brasileiro, incluindo Porto Belo-SC, onde os peixes representaram 65,96\% da fauna acompanhante amostrada. Essa dominância pode estar associada a diversos fatores como temperatura, salinidade, sedimento, correntes, profundidade, estação do ano e disponibilidade de alimentos em cada área de pesca (Carranza-Fraser \& Grande 1982, Haimovici et al. 1996, Branco \& Verani 2006b, Souza et al. 2008, Freitas et al. 2011).

Entre os peixes demersais, a família Sciaenidae é o recurso mais capturado pela frota arrasteira de camarão sete-barbas na região Sudeste-Sul (Graça-Lopes et al. 2002, Rocha \& RossiWongtschowski 1998; Branco 2005, Branco \& Verani 2006a, b, Schwarz Junior et al. 2006, Souza et al. 2008, Bernardes Júnior et al. 2011, Cattani et al. 2011, Freitas et al. 2011), com as maiores taxas de captura incidindo sobre Stellifer rastrifer, S. brasiliensis, S. stellifer, Paralonchurus brasiliensis, Micropogonias furnieri, Larimus breviceps, Isopisthus parvipinnis, Cynoscion jamaicensis, Pellona harroweri e Menticirrhus americanus, que estão entre as 12 espécies de maior abundância e biomassa coletadas em Porto Belo-SC. 


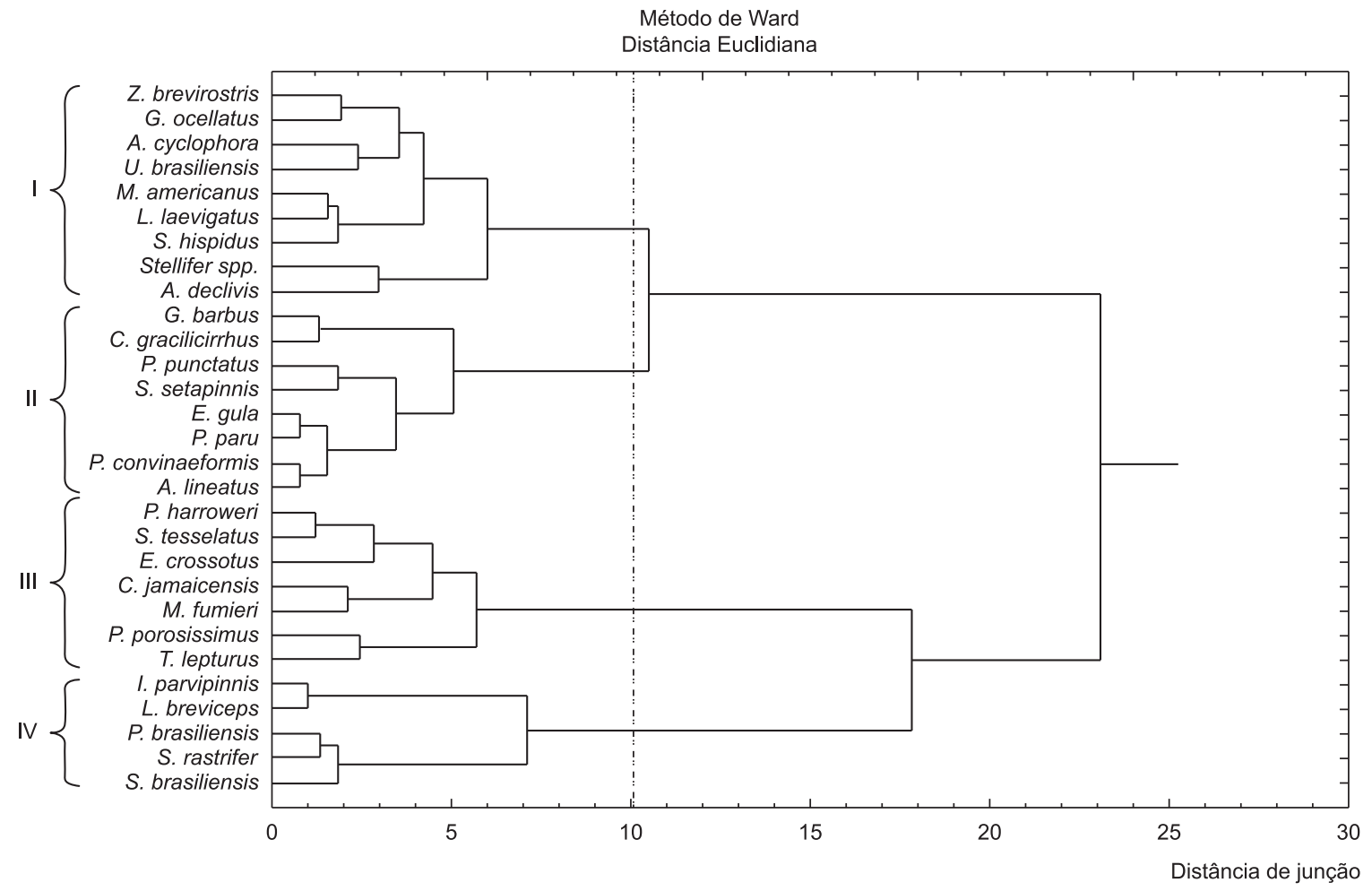

Figura 4. Dendrograma do agrupamento das 29 espécies da ictiofauna acompanhante capturadas em mais de quatro coletas anuais realizadas na pesca de arrasto do camarão sete-barbas (Xiphopenaeus kroyeri) em Porto Belo, SC.

Figure 4. Dendrogram of the grouping of the 29 captured species of ichthyofauna accompanying in more than four carried through annual collections in artisanal fishing for the sea-bob shrimp (Xiphopenaeus kroyeri), in Porto Belo, SC, Brazil.

É possível que esses peixes procurem as áreas onde se encontram os camarões Penaeoidea para obtenção de alimentos e ou em busca de fatores abióticos favoráveis, principalmente o sedimento e a profundidade, determinantes nas relações ecológicas entre esses grupos (Souza et al. 2008).

O número de espécies da ictiofauna acompanhante em cada arrasto variou desde uma nos $20 \mathrm{~m}$ durante o inverno até 25 , em $10 \mathrm{~m}$, no verão, e cerca de $80 \%$ das capturas de Porto Belo foram constituídas por espécies ocasionais e pouco frequentes, se aproximando de Branco \& Verani (2006b) na Armação de Itapocoroy, Penha, SC, que justifica esse elevado número pela ocorrência de espécies visitantes ou em trânsito, encontradas também em regiões mais profundas e estuários (Coelho et al. 1986, Branco \& Verani 2006b, Bernardes Júnior et al. 2011). Portanto, apenas uma parcela pequena de espécies da ictiofauna acompanhante, domina em abundância e biomassa, onde a maioria é de ocorrência rara (Coelho et al. 1986, Branco \& Verani 2006b, Bernardes Júnior et al. 2011).

Geralmente as flutuações espaçotemporais na abundância acompanham as variações da CPUE, sendo esse, o índice mais adequado para o monitoramento da pesca, portanto, sua alteração, significa alterações temporais na abundância de peixes (Branco et al. 2005). As médias sazonais mais elevadas em abundância e CPUE da ictiofauna acompanhante foram obtidas na primavera e outono, no litoral do Rio Grande do Sul (Haimovici et al. 1996), verão e inverno na Armação de Itapocoroy/Penha (Branco \& Verani 2006b) e nos meses mais quentes, na plataforma continental do Paraná (Cattani et al. 2011).

$\mathrm{Na}$ área de estudo, as maiores abundâncias e CPUE ocorreram no verão e inverno, especialmente nos $10 \mathrm{~m}$, corroborando com os estudos de Souza et al. (2008) no litoral norte São Paulo, associando dentre outros fatores, a retração da ACAS e a ampliação das águas costeiras nesse último período, diminuindo a salinidade, elevando a temperatura e disponibilidade de alimentos em profundidades menores, além da instabilidade do fundo mais raso, arenosílticoargilosos e remexidos, causada pela passagem de correntes frias que expõem os camarões e atraem os peixes Sciaenidae, abundantes nessas profundidades. Assim, pode-se supor que condições semelhantes possam ser encontradas em Porto Belo, SC.

Portanto, as variações sazonais na abundância e CPUE, podem estar relacionadas aos ciclos reprodutivos das espécies, relações tróficas, hidrodinâmica, e instabilidade da região costeira, com consequentes alterações qualiquantitativas em seus componentes abióticos e bióticos (Giannini \& Paiva-Filho 1995, Rossi-Wongtschowski et al. 2008, Soares-Gomes \& Figueiredo 2002, Schwarz Junior et al. 2006, Branco \& Verani 2006b, RossiWongtschowski et al. 2008, Souza et al. 2008, Bernardes Júnior et al. 2011).

Os valores do índice de diversidade (H') registrados no presente estudo oscilaram em torno dos obtidos em Penha 0,92 e 3,43 (Branco \& Verani 2006b), 0,39 e 2,16 (Bail \& Branco 2003) e Barra do Sul 1,04 e 1,98 (Bernardes Júnior et al. 2011). Essa diversidade pode ser influenciada de modo negativo, pela presença de poucas espécies dominantes (Pires-Vanin 1977, Giannini \& Paiva-Filho 1995), locais inadequados para a desova, falta de refúgio e alimentos para jovens, presença de espécies predadoras e forrageiras, além de ações antrópicas, como o esforço de pesca (Washington 1984, Cecílio et al. 1997, Bernardes Júnior et al. 2011).

O índice de equitabilidae ( $\mathrm{J}$ '), também se manteve consistente com os autores supracitados, onde há dominância de poucas espécies (Ansari et al. 1995, Branco \& Verani 2006b) e, como a média anual manteve-se acima de 0,5 esse índice sugere para a área de estudo, a 
distribuição espaçotemporal, relativamente uniforme entre as espécies (Bernardes Júnior et al. 2011).

A análise de Cluster originou quatro agrupamentos obedecendo à frequência de ocorrência das espécies registradas, sendo que um grupo pequeno e dominante reuniu as espécies de Sciaenidae com maior abundância e biomassa, sendo quatro foram frequentes, Isopisthus parvipinnis, Larimus breviceps, Stellifer brasiliensis e $S$. rastrifer e uma, muito frequente, a Paralonchurus brasiliensis. Os dois maiores agrupamentos reuniram as espécies pouco numerosas e maioria pouco frequentes, enquanto que o terceiro reuniu seis espécies entre as 12 mais abundantes, sendo a maioria frequentes. Esses resultados são corroborados por diversos autores, que obtiveram poucas espécies dominantes para o litoral brasileiro, tanto em abundância quanto em biomassa (Haimovici et al. 1998, Araújo et al. 1998, Rocha \& RossiWongtschowski 1998, Branco \& Verani 2006b, Rodrigues et al. 2007, Souza et al. 2008, Bernardes Júnior et al. 2011).

\section{Conclusão}

Na pesca de arrasto do camarão sete-barbas em Porto Belo, SC, raramente foram capturados peixes de grande porte e normalmente, descarta-se toda a fauna acompanhante, incluindo espécies de valor comercial como cangoás, pescadas, corvina, maria-luiza e oveva, dentre outras. Essa prática frequente, pode alterar a estrutura desse ecossistema costeiro, colocando em risco a sustentabilidade da espécie-alvo, fauna acompanhante e toda a comunidade biológica associada.

Portanto, diante dos resultados obtidos, recomenda-se em Porto Belo/SC e região, a adoção de políticas públicas, que visem o estudo e manejo dos recursos pesqueiros para não comprometer o futuro da pesca artesanal, desse histórico município de Santa Catarina.

\section{Agradecimentos}

A todas as pessoas que colaboraram no levantamento de dados, especialmente àquelas do laboratório de biologia do CTTMar-Univali pelo auxílio na identificação, quantificação e registro das biomassas dos exemplares capturados, fundamental para a elaboração do presente artigo. Ao CNPq pelas bolsas de produtividade de J. O. B. e E. B.

\section{Referências Bibliográficas}

ALVERSON, D.L., FREEBERG, M.H., POPE, J.G., MURAWSKI, S.A. 1994. A global assessment of fisheries bycatch and discards. FAO Fish. Tech. Pap. 339:1-233.

ANSARI, Z.A., CHATTERFI, A., INGOLE, B.S., SREEPADA, R.A., RIVONKAR, C.U. \& PARULEKAR, A.H. 1995. Community structure seasonal variation of on inshore demersal fish community at Goa, west coast of India. Estuar. Coast. Shelf Sci. 41:593-610. http://dx.doi. org/10.1016/0272-7714(95)90029-2

ARAÚJO, F.G., CRUZ-FILHO, A.G., AZEVEDO, M.C.C. \& SANTOS, A.C.A. 1998. Estrutura da comunidade de peixes demersais da Baía de Sepetiba, RJ. Rev. Bras. Biol. 58(3):417-430. http://dx.doi.org/10.1590/ S0034-71081998000300007

BAIL, G.C., BRANCO, J.O., FREITAS JÚNIOR, F., LUNARDONBRANCO, M.J. \& BRAUN, J.R.R. 2009. Fauna acompanhante do camarão sete barbas, na Foz do Rio Itajaí-Açú e sua contribuição na diversidade de crustáceos e peixes do ecossistema Saco da Fazenda. In Estuário do Rio Itajaí-Açú, Santa Catarina: caracterização ambiental e alterações antrópicas (J.O. Branco, M.J. Lunardon-Branco \& V.R. Bellotto). Editora da UNIVALI, Itajaí, p.284-312.

BAIL, G. C. \& BRANCO, J.O. 2003. Ocorrência, abundância e diversidade da Ictiofauna na pesca do camarão sete-barbas, na região de Penha, SC. Notas Téc. FACIMAR 7:73-82.
BERNARDES JÚNIOR, J.J., RODRIGUES FILHO, J., BRANCO, J.O. \& VERANI, J.R. 2011. Spatiotemporal variations of the ichthyofaunal structure accompanying the seabob shrimp, Xiphopenaeus kroyeri (Crustacea: Penaeidae), fishery in important fishery areas of the Santa Catarina shore, Brazil. Zoologia 28(2):151-164.

BRANCO, J.O. 2005. Biologia e pesca do camarão sete-barbas Xiphopenaeus kroyeri (Heller) (Crustacea, Penaeidae), na Armação do Itapocoroy, Penha, SC. Rev. Bras. Zool. 22(4):1050-1062. http://dx.doi.org/10.1590/ S0101-81752005000400034

BRANCO, J.O., BAIL, G.C., VERANI, J. R. \& MARENZI, A.W.C. 2006. Aspectos sócio-econômicos da pesca artesanal do camarão sete-barbas (Xiphopenaeus kroyeri), na região de Penha, SC. In Bases ecológicas para um desenvolvimento sustentável: estudos de caso em Penha, SC (J.O. Branco \& A.W.C. Marenzi). Editora da UNIVALI, Itajaí, p.253-268.

BRANCO, J.O., LUNARDON-BRANCO, M.J. \& VERANI, J.R. 2005. Aspectos biológicos e pesqueiros de Paralonchurus brasiliensis Steindachner, (Pisces, Sciaenidae), na Armação do Itapocoroy, Penha, Santa Catarina, Brasil. Rev. Bras. Zool. 22(4):1063-1071. http://dx.doi. org/10.1590/S0101-81752005000400035

BRANCO, J.O. \& VERANI, J.R. 2006a. Pesca do camarão sete-barbas e sua fauna acompanhante, na Armação do Itapocoroy, Penha, SC. In Bases ecológicas para um desenvolvimento sustentável: estudos de caso em Penha, SC (J.O. Branco \& A.W.C. Marenzi). Editora da UNIVALI, Itajaí, p.153-170.

BRANCO, J.O. \& VERANI, J.R. 2006b. Análise quali-quantitativa da ictiofauna acompanhante na pesca do camarão sete-barbas, na Armação do Itapocoroy, Penha, Santa Catarina. Rev. Bras. Zool. 23(2):381-391. http://dx.doi.org/10.1590/S0101-81752006000200011

BRASIL. Ministério da Pesca e da Aquicultura - MPA. Informações e Estatística. http://www.mpa.gov.br (último acesso em: 18/12/2011).

CARRANZA-FRASER, J. \& GRANDE, J.M. 1982. Experiencia de México en el aprovechamiento de la fauna de acompañamiento del camarón. Proc. Gulf Caribb. Fish. Inst. USA 39:109-111.

CARVALHO, J.L.B., SCHETTINI, C.A. F., RIBAS, T.M. 1998. Estrutura Termohalina do Litoral Centro-Norte Catarinense. Notas Téc. FACIMAR 2:181-197.

CATTANI, A.P., SANTOS, L.O., SPACH, H.L., BUDEL, B.R., GONDIM GUANAIS, J.H.D. 2011. Avaliação da ictiofauna da fauna acompanhante da pesca do camarão sete-barbas do município de Pontal do Paraná, litoral do Paraná, Brasil. Bol. Inst. Pesca 37(2):247-260.

CECÍLIO, E.B., AGOSTINHO, A.A., JÚLIO JUNIOR, H.F., PAVANELLI, C.S. 1997. Colonização ictiofaunística do reservatório de Itaipu e áreas adjacentes. Rev. Bras. Zool. 14(1):1-14.

COELHO, J.A.P., PUZZI, A., GRAÇA-LOPES, R., RODRIGUES, E.S., PRIETO JUNIOR, O. 1986. Análise da rejeição de peixes na pesca artesanal dirigida ao camarão sete-barbas (Xiphopenaeus kroyeri) no litoral do Estado de São Paulo. Bol. Inst. Pesca 13(2):51-61.

COLVOCORESSES, J.A. \& MUSICK, J.A. 1984. Species associations and community composition of Middle Atlantic Bight continental shelf demersal fishes. Fish. Bull. (U.S.) 82:295-313.

DIEGUES, A.C.S. 1983. Pescadores, camponeses e trabalhadores do mar. Editora Ática, São Paulo, 559p.

EMPRESA DE PESQUISA AGROPECUÁRIA E EXTENSÃO RURAL DE SANTA CATARINA. Centro de Socioeconomia e Planejamento Agrícola - Epagri/Cepa. Síntese Anual da Agricultura de Santa Catarina 2009-2010. http://cepa.epagri.sc.gov.br/Publicacoes/ Sintese_2010/(último acesso em 18/12/2011).

FIGUEIREDO, J.L. 1977. Manual de peixes marinhos do Sudeste do Brasil. I. Introdução. Cações, raias e quimeras. Museu de Zoologia, Universidade se São Paulo, 104p.

FIGUEIREDO, J.L. \& MENEZES, N.A. 1978. Manual de peixes marinhos do sudeste do Brasil. II. Teleostei (1). Museu de Zoologia, Universidade de São Paulo, 110p.

FIGUEIREDO, J.L. 1980. Manual de peixes marinhos do sudeste do Brasil. III. Teleostei (2). Museu de Zoologia, Universidade de São Paulo, 90p. 
FIGUEIREDO, J.L. \& MENEZES, N.A. 2000. Manual de peixes marinhos do Sudeste do Brasil: VI. Teleostei (5). Museu de Zoologia, Universidade de São Paulo, 116p.

FISHBASE. 2011. www.fishbase.org/ (último acesso em 11/12/2011).

FREITAS, M.O., SPACH, H.L., HOSTIM-SILVA, M. 2011. Variação espaçotemporal da assembleia de peixes demersais em uma área de pesca do camarão sete-barbas no sul do Brasil. Neotrop. Biol. Conserv. 6(1):44-54. http://dx.doi.org/10.4013/nbc.2011.61.06

GIANNINI, R. \& PAIVA-FILHO, A.M. 1995. Distribuição temporal, espacial e bioecologia do cangoá, Stellifer brasiliensis (Teleostei: Sciaenidae), na Baía de Santos, São Paulo, Brasil. Arq. Ciênc. Mar 29(1-2):5-15.

GRAÇA-LOPES, R., SEVERINO-RODRIGUES, E., PUZZI, A., PITA, J.B., COELHO, J.A.P., FREITAS, M.L. 1993. Levantamento ictiofaunístico em um ponto fixo na Baía de Santos, Estado de São Paulo, Brasil. Bol. Inst. Pesca 20:7-20.

GRAÇA-LOPES, R., TOMÁS, A.R.G., TUTUI, S.L.S., SEVERINO RODRIGUES, E., PUZZI, A. 2002. Fauna acompanhante da pesca camaroeira no litoral do estado de São Paulo, Brasil. Bol. Inst. Pesca 28(2):173-188.

HAIMOVICI, M., CASTELLO, J.P., VOOREN, C.M. 1998. Pescarias. In Os Ecossistemas Costeiro e Marinho do Extremo Sul do Brasil (U. Seeliger, C. Odebrecht \& J.P. Castelllo). Editora Ecoscientia, Rio Grande, p.206-218.

HAIMOVICI, M., MARTINS, A. S., VIEIRA, P. C. 1996. Distribuição e abundância de peixes teleósteos demersais sobre a plataforma continental do sul do Brasil. Rev. Bras. Biol. 56(1):27-50.

INSTITUTO BRASILEIRO DE GEOGRAFIA E ESTATÍSTICA - IBGE. http://www.ibge.gov.br (último acesso em 18/12/2011).

LUDWIG, J.A. \& REYNOLDS, J.F. 1988. Statistical ecology: a primer on methods and computing. John Wiley and Sons New York, 338p.

MATSUURA, Y. 1986. Contribuição ao estudo da estrutura oceanográfica da Região Sudeste entre Cabo Frio (RJ) e Cabo de Santa Marta Grande (SC). Cienc. Cult. 38(8):1439-1450.

MENEZES, N.A. \& FIGUEIREDO, J.L. 1980. Manual de peixes marinhos do sudeste do Brasil. IV. Teleostei (3). Museu de Zoologia, Universidade de São Paulo, 96p.

MENEZES, N.A. \&. FIGUEIREDO, J.L. 1985. Manual de peixes marinhos do sudeste do Brasil. V. Teleostei (4). Museu de Zoologia, Universidade de São Paulo, 105p.

PINHEIRO, H.T. \& MARTINS, A.S. 2009. Estudo comparativo da captura artesanal do camarão sete-barbas e sua fauna acompanhante em duas áreas de pesca do litoral do estado do Espírito Santo, Brasil. Bol. Inst. Pesca 35(2):215-225.
PIRES-VANIN, A.M.S. 1977. Aspectos da fauna de isópoda (crustacea peracarida) das zonas litoral e infralitoral de fundos duros da enseada do Flamengo, Ubatuba, SP. Tese de Doutorado, Universidade de São Paulo, São Paulo, 83p.

ROCHA, G.R.A. \& ROSSI-WONGTSCHOWSKI, C.L.D.B. 1998. Demersal fish community on the inner shelf of Ubatuba, southestern Brazil. Rev. Bras. Oceanogr. 46:93-109.

ROCHA, I. P. \& ROCHA, D. M. 2010. Panorama da Produção Mundial e Brasileira de Pescados, com ênfase para o Segmento da Aquicultura. http://www.abccam.com.br (último acesso em18/12/2011).

RODRIGUES, C., LAVRADO, H.P., FALCÃO, A.P.C., SILVA, S.H.G. 2007. Distribuição da ictiofauna capturada em arrastos de fundo na Baía de Guanabara - Rio de Janeiro, Brasil. Arq. Mus. Nac. 65(2):199-210.

ROSSI-WONGTSCHOWSKI, C.L.D.B., SOARES, L.S.H., MUTO, E.Y. 2008 Ictiofauna. In Oceanografia de um Ecossistema Subtropical - Plataforma de São Sebastião, SP (A.M.S. Pires-Vanin). Editora da Universidade de São Paulo, São Paulo, p.381-404.

SCHWARZ JUNIOR, R., FRANCO, A.C.N.P., SPACH, H.L., SARPEDONTI, V., PICHLER, H.A., NOGUEIRA DE QUEIROZ, G.M.L. 2006. Composição e estrutura da ictiofauna demersal na Baía dos Pinheiros, Paraná. Braz. J. Aquat. Sci. Tech. 10(1):27-39.

SLAVIN, J.W. 1983. Utilización de la pesca acampañante del camarón. In Pesca acompañante del camarón - un regalo del mar - informe de uma consulta técnica sobre utilización de la pesca acompañante del camarón. Georgetown Guyana, Otawa, Ont. CIID, p.67-71. Pmid:6224019.

SOARES, L.H. 1978. Estudo dos Bothidae, Cynoglossidae e Soleidae capturados nos bancos de camarão, no Estado do Rio Grande do Norte - Brasil. Bol. Dep. Oceanogr. Limnol. Cent. Biocienc. Univ. Fed. Rio Gd. Norte 6:16-27.

SOARES-GOMES, A. \& FIGUEIREDO, A.G. 2002. O ambiente marinho. In PEREIRA, R.C., SOARES-GOMES, A. Biologia Marinha. Interciência, Rio de Janeiro, p.1-33.

SOKAL, R.R. \& ROHLF, F.J. 1995. Biometry; the principles and practice of statistics in biological research. 3th ed. W. H. Freeman and Co., San Francisco, 887p.

SOUZA, U. P., COSTA, R. C. D., MARTINS, I. A., FRANSOZO, A. 2008. Associações entre as biomassas de peixes Sciaenidae (Teleostei: Perciformes) e de camarões Penaeoidea (Decapoda: Dendrobranchiata) no litoral norte do Estado de São Paulo. Biota Neotrop. 8(1):83-92. http:// dx.doi.org/10.1590/S1676-06032008000100011

TOPP, R.W. \& HOFF JUNIOR, F.H. 1972. Flatfishes (Pleuronectiformes). Florida Department of Natural Resources, Memoirs of the Hourglass Cruises, $135 \mathrm{p}$.

WASHINGTON, H.G. 1984. Diversity, biotic and similaraty indices: a rewiew with special relevance to aquatic ecosystems. Water Res. 18:653-694. http://dx.doi.org/10.1016/0043-1354(84)90164-7

Recebido em 23/05/2012 Versão reformulada recebida em 17/12/2012 Publicado em 04/01/2013 